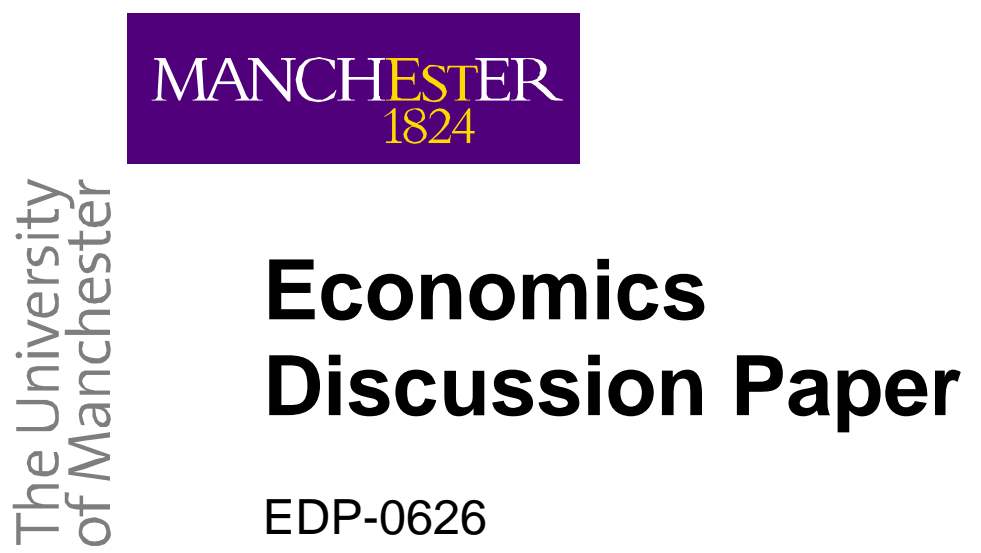

\title{
Volatility-Induced Financial Growth
}

\author{
Michael A.H. Dempster, Igor V. Evstigneev and \\ Klaus R. Schenk-Hoppé
}

October 2006

Correspondence email: igor.evstigneev@man.ac.uk

School of Social Sciences,

The University of Manchester

Oxford Road

Manchester M13 9PL

United Kingdom 


\title{
VOLATILITY-INDUCED FINANCIAL GROWTH*
}

\author{
Michael A.H. Dempster ${ }^{a}$, Igor V. Evstigneev ${ }^{b}$ and \\ Klaus R. Schenk-Hoppé ${ }^{c}$
}

\begin{abstract}
We show that the volatility of a price process, which is usually regarded as an impediment to financial growth, can serve as an endogenous factor in its acceleration.
\end{abstract}

${ }^{*}$ We are indebted to numerous colleagues who had enough patience to participate in our "intuition tests," the results of which are discussed in Section 4 of this paper. Our comparative analysis of volatility-induced growth and arbitrage (also contained in Section 4) was motivated by Walter Schachermayer's comments at a conference on Mathematical Finance in Paris in 2003. Albert N. Shiryaev deserves our thanks for a helpful discussion of some mathematical questions related to this work.

Financial support by the Swiss National Centre of Competence in Research "Financial Valuation and Risk Management" (NCCR FINRISK) is gratefully acknowledged.

${ }^{a}$ Centre for Financial Research, Judge Business School, University of Cambridge, Trumpington Street, Cambridge, CB2 1AG, United Kingdom (e-mail: mahd2@cam.ac.uk).

$b$ School of Economic Studies, University of Manchester, Oxford Road, Manchester, M13 9PL, United Kingdom (e-mail: igor.evstigneev@manchester.ac.uk).

${ }^{c}$ Leeds University Business School and School of Mathematics, University of Leeds, Leeds, LS2 9JT, United Kingdom (e-mail: k.r.schenk-hoppe@leeds.ac.uk).

This paper is a substantially revised and extended version of Working Paper No. 10/2004, Centre for Financial Research, Judge Institute of Management, University of Cambridge, September 2004. 


\section{Introduction}

Can volatility, which is present in virtually every financial market and usually thought of in terms of a risky investment's downside, serve as an "engine" for financial growth? Paradoxically, the answer to this question turns out to be positive.

To demonstrate this paradox, we examine the long-run performance of constant proportions investment strategies in a securities market. Such strategies prescribe rebalancing the investor's portfolio, depending on price fluctuations, so as to keep fixed proportions of wealth in all the portfolio positions. Assume that asset returns form a stationary ergodic process and asset prices grow (or decrease) at a common asymptotic rate $\rho$. It is shown in this paper that if an investor employs any constant proportions strategy, then the value of his/her portfolio grows almost surely at a rate strictly greater than $\rho$, provided that the investment proportions are strictly positive and the stochastic price process is in a sense non-degenerate. The very mild assumption of non-degeneracy we impose requires at least some randomness, or volatility, of the price process. If this assumption is violated, then the market is essentially deterministic and the result ceases to hold. Thus, in the present context, the price volatility may be viewed as an endogenous source of acceleration of financial growth. This phenomenon might seem counterintuitive, especially in stationary markets $[7,10]$, where the asset prices themselves, and not only their returns, are stationary. In this case, $\rho=0$, i.e. each asset grows at zero rate, while any constant proportions strategy exhibits growth at a strictly positive exponential rate with probability one!

To begin with, we focus on the case where all the assets have the same growth rate $\rho$. The results are then extended to a model with different growth rates $\rho^{1}, \ldots, \rho^{K}$. In this setting, a constant proportions strategy with proportions $\lambda^{1}>0, \ldots, \lambda^{K}>0$ grows almost surely at a rate strictly greater than $\sum_{k} \lambda^{k} \rho^{k}$ (see Theorem 1 in Section 2).

The phenomenon highlighted in this paper has been mentioned in the literature, but its analysis has been restricted to examples involving specialized models. The terms "excess growth" (Fernholz and Shay [11]) and "volatility pumping" (Luenberger [21]) have been used to name similar effects to those discussed here. Cover [6] used the mechanism of volatility pumping in the construction of universal portfolios. These ideas have been discussed in connection with financial market data in Mulvey [24], Mulvey and Ziemba [25], and Dries et al. [8]. Such questions have typically been studied in the context of maximization of expected logarithmic utilities - "log-optimal 
investments" (Kelly [18], Breiman [5], Algoet and Cover [2], MacLean at al. [22], Hakansson and Ziemba [13], Li [20], Aurell et al. [3], and others). In this paper we ignore questions of optimality of trading strategies and do not use the related notion of expected utility involved in optimality criteria. ${ }^{1}$

Constant proportions strategies play an important role in various practical financial computations, see e.g. Perold and Sharpe [26]. The assumption of stationarity of asset returns is widely accepted in financial theory and practice allowing, as it does, expected exponential price growth and mean reversion, volatility clustering and very general intertemporal dependence, such as long memory effects, of returns. However, no general results justifying and explaining the fact of volatility-induced growth have been established up to now. In spite of the fundamental importance and generality of this fact, no results pertaining to an arbitrary constant proportions strategy (regardless of its optimality) and any securities market with stationary non-degenerate asset returns have been available in the literature. The purpose of this paper is to fill this gap.

Most of our results are rather easy consequences of some general mathematical facts, and the mathematical aspects do not play a crucial role. The main contribution of the present work is that we pose and analyze a number of questions that have not been systematically analyzed before. These questions are especially interesting because the common intuition currently prevailing in the mathematical finance community suggests wrong answers to them (see the discussion in Section 4). Therefore it is important to clarify the picture in order to reveal and correct misconceptions. This is a central goal in this study.

The paper is organized as follows. In Section 2 we describe the model, formulate the assumptions and state the main results. Section 3 contains proofs of the results and a discussion of their intuitive meaning. In Sections 4 and 5 we analyze the phenomenon of volatility-induced growth from various angles, focussing primarily on the case of stationary prices. We answer a number of questions arising naturally in connection with the theory developed. In Section 6, we show how this theory can be extended to markets with small transaction costs. Section 7 analyzes an example in which estimates for the size of transaction cost rates allowing volatility-induced

\footnotetext{
${ }^{1}$ In connection with the discussion of relevant literature, we can mention a strand of publications dealing with Parrondo games [14]. Models considered in those publications are based on the analysis of lotteries whose odds depend on the investor's wealth. It is pointed out that losing lotteries, being played in a randomized alternating order, can become winning. In spite of some similarity, there are no obvious direct links between this phenomenon and that studied in the present paper.
} 
growth can be established.

\section{The model and the main results}

Consider a financial market with $K \geq 2$ securities (assets). Let $S_{t}:=$ $\left(S_{t}^{1}, \ldots, S_{t}^{K}\right)$ denote the vector of security prices at time $t=0,1,2, \ldots$ Assume that $S_{t}^{k}>0$ for each $t$ and $k$, and define by

$$
R_{t}^{k}:=\frac{S_{t}^{k}}{S_{t-1}^{k}}(k=1,2, \ldots, K, t=1,2, \ldots)
$$

the (gross) return on asset $k$ over the time period $(t-1, t]$. Let $R_{t}:=$ $\left(R_{t}^{1}, \ldots, R_{t}^{K}\right)$. At each time period $t$, an investor chooses a portfolio $h_{t}=\left(h_{t}^{1}\right.$, $\left.\ldots, h_{t}^{K}\right)$, where $h_{t}^{k}$ is the number of units of asset $k$ in the portfolio $h_{t}$. Generally, $h_{t}$ might depend on the observed values of the price vectors $S_{0}, S_{1}, \ldots, S_{t}$. A sequence $H=\left(h_{0}, h_{1}, \ldots\right)$ specifying a portfolio $h_{t}=h_{t}\left(S_{0}, \ldots, S_{t}\right)$ at each time $t$ as a measurable function of $S_{0}, S_{1}, \ldots, S_{t}$ is called a trading strategy. If not otherwise stated, we will consider only those trading strategies for which $h_{t}^{k} \geq 0$, thus excluding short sales of assets $\left(h_{t}^{k}\right.$ can take on all non-negative real values).

One can specify trading strategies in terms of investment proportions (or portfolio weights). Suppose that for each $t=1,2, \ldots$, we are given a vector $\lambda_{t}=\left(\lambda_{t}^{1}, \ldots, \lambda_{t}^{K}\right)$ in the unit simplex

$$
\Delta:=\left\{\lambda=\left(\lambda^{1}, \ldots, \lambda^{K}\right): \lambda^{k} \geq 0, \sum_{k=1}^{K} \lambda^{k}=1\right\} .
$$

The vector $\lambda_{t}$ is assumed to be a measurable function of $S_{0}, \ldots, S_{t}$. Given an initial portfolio $h_{0}$ (specified by a non-negative non-zero vector), we can construct a trading strategy $H$ recursively by the formula

$$
h_{t}^{k}=\lambda_{t}^{k} S_{t} h_{t-1} / S_{t}^{k}(k=1,2, \ldots, K, t=1,2, \ldots) .
$$

Here the scalar product $S_{t} h_{t-1}=\sum_{k=1}^{K} S_{t}^{k} h_{t-1}^{k}$ expresses the value of the portfolio $h_{t-1}$ in terms of the prices $S_{t}^{k}$ at time $t$. An investor following the strategy (2) rebalances (without transaction costs) the portfolio $h_{t-1}$ at time $t$ so that the available wealth $S_{t} h_{t-1}$ is distributed across the assets $k=1,2, \ldots, K$ according to the proportions $\lambda_{t}^{1}, \ldots, \lambda_{t}^{K}$. It is immediate from (2) that

$$
S_{t} h_{t}=S_{t} h_{t-1}, t=1,2, \ldots,
$$


i.e., the strategy $H$ is self-financing. If a strategy is self-financing, then the relations (2) and

$$
S_{t}^{k} h_{t}^{k}=\lambda_{t}^{k} S_{t} h_{t}, t=1,2, \ldots,
$$

are equivalent. If the vectors of proportions $\lambda_{t}$ are fixed (do not depend on time and on the price process), i.e. $\lambda_{t}=\lambda=\left(\lambda^{1}, \ldots, \lambda^{K}\right) \in \Delta$, then the strategy $H$ defined recursively by

$$
h_{t}^{k}=\lambda^{k} S_{t} h_{t-1} / S_{t}^{k}(k=1,2, \ldots, K, t=1,2, \ldots)
$$

is called a constant proportions strategy (or a fixed-mix strategy) with vector of proportions $\lambda=\left(\lambda^{1}, \ldots, \lambda^{K}\right)$. If $\lambda^{k}>0$ for each $k$, then $H$ is said to be completely mixed.

We will assume that the price vectors $S_{t}$, and hence the return vectors $R_{t}$, are random, i.e., they change in time as stochastic processes. Then the trading strategy $h_{t}, t=0,1,2, \ldots$, generated by the investment rule (2) and the value $V_{t}=S_{t} h_{t}, t=0,1,2, \ldots$, of the portfolio $h_{t}$ are stochastic processes as well. We are interested in the asymptotic behavior of $V_{t}$ as $t \rightarrow \infty$ for constant proportions strategies.

We will assume:

(R) The vector stochastic process $R_{t}, t=1,2, \ldots$, is stationary and ergodic. The expected values $E\left|\ln R_{t}^{k}\right|, k=1,2, \ldots, K$, are finite.

Recall that a stochastic process $R_{1}, R_{2}, \ldots$ is called stationary if, for any $m=0,1,2, \ldots$ and any measurable function $\phi\left(x_{0}, x_{1}, \ldots, x_{m}\right)$, the distribution of the random variable $\phi_{t}:=\phi\left(R_{t}, R_{t+1}, \ldots, R_{t+m}\right)(t=0,1, \ldots)$ does not depend on $t$. According to this definition, all probabilistic characteristics of the process $R_{t}$ are time-invariant. If $R_{t}$ is stationary, then for any measurable function $\phi$ for which $E\left|\phi\left(R_{t}, R_{t+1}, \ldots, R_{t+m}\right)\right|<\infty$, the averages

$$
\frac{\phi_{1}+\ldots+\phi_{t}}{t}
$$

converge almost surely (a.s.) as $t \rightarrow \infty$ (Birkhoff's ergodic theorem-see, e.g., Billingsley [4]). If the limit of all averages of the form (6) is non-random (equal to a constant a.s.), then the process $R_{t}$ is called ergodic. In this case, the above limit is equal a.s. to the expectation $E \phi_{t}$, which does not depend on $t$ by virtue of stationarity of $R_{t}$.

An example of a stationary ergodic process is a sequence of independent identically distributed (i.i.d.) random variables. To avoid misunderstandings, we emphasize that Brownian motion and a random walk are not stationary. According to the conventional probabilistic terminology, these Markov processes are (time) homogeneous. 
We have $S_{t}^{k}=S_{0}^{k} R_{1}^{k} \ldots R_{t}^{k}$, where (according to $(\mathbf{R})$ ) the random sequence $R_{t}^{k}$ is stationary. This assumption on the structure of the price process is a fundamental hypothesis commonly accepted in finance. Moreover, it is quite often assumed that the random variables $R_{t}^{k}, t=1,2, \ldots$ are independent, i.e., the price process $S_{t}^{k}$ forms a geometric random walk. This postulate, which is much stronger than the hypothesis of stationarity of $R_{t}^{k}$, lies at the heart of the classical theory of asset pricing (Black, Scholes, Merton), see e.g. Luenberger [21].

By virtue of Birkhoff's ergodic theorem, we have

$$
\lim _{t \rightarrow \infty} \frac{1}{t} \ln S_{t}^{k}=\lim _{t \rightarrow \infty} \frac{1}{t} \sum_{j=1}^{t} \ln R_{j}^{k}=E \ln R_{t}^{k}(\text { a.s. })
$$

for each $k=1,2, \ldots, K$. This means that the price of each asset $k$ has almost surely a well-defined and finite (asymptotic, exponential) growth rate, which turns out to be equal a.s. to the expectation $\rho^{k}:=E \ln R_{t}^{k}$, the drift of this asset's price. The drift can be positive, zero or negative. It does not depend on $t$ in view of the stationarity of $R_{t}$. Let $H=\left(h_{0}, h_{1}, \ldots\right)$ be a trading strategy. If the limit

$$
\lim _{t \rightarrow \infty} \frac{1}{t} \ln \left(S_{t} h_{t}\right)
$$

exists, it is called the (asymptotic, exponential) growth rate of the strategy $H$.

We now formulate central results of this paper-Theorems 1 and 2. In these theorems, $H=\left(h_{0}, h_{1}, \ldots\right)$ is a constant proportions strategy with some vector of proportions $\lambda=\left(\lambda^{1}, \ldots, \lambda^{K}\right) \in \Delta$ and a non-zero initial portfolio $h_{0} \geq 0$. In Theorems 1 and 2 , we assume that the following condition holds:

(V) With strictly positive probability,

$$
S_{t}^{k} / S_{t}^{m} \neq S_{t-1}^{k} / S_{t-1}^{m} \text { for some } 1 \leq k, m \leq K \text { and } t \geq 1 .
$$

Theorem 1. If all the coordinates $\lambda^{k}$ of the vector $\lambda$ are strictly positive, i.e. the strategy $H$ is completely mixed, then the growth rate of the strategy $H$ is almost surely equal to a constant which is strictly greater than $\sum_{k} \lambda^{k} \rho^{k}$, where $\rho^{k}$ is the drift of asset $k$.

Condition $(\mathbf{V})$ is a very mild assumption of volatility of the price process. This condition does not hold if and only if, with probability one, the ratio $S_{t}^{k} / S_{t}^{m}$ of the prices of any two assets $k$ and $m$ does not depend on $t$. Thus condition ( $\mathbf{V})$ fails to hold if and only if the relative prices of the assets are constant in time (a.s.). 
We are primarily interested in the situation when all the assets under consideration have the same drift and hence a.s. the same asymptotic growth rate:

(R1) There exists a number $\rho$ such that, for each $k=1, \ldots, K$, we have $E \ln R_{t}^{k}=\rho$.

Assumption (R1) allows one to concentrate, for example, on those assets in the market whose prices grow at the maximum rate. One may think that all the others, growing more slowly, will eventually be driven out of the market. As long as we deal with an infinite time horizon, we may exclude such assets from consideration.

From Theorem 1, we immediately obtain the following result.

Theorem 2. Under assumption ( $\boldsymbol{R} 1)$, the growth rate of the strategy $H$ is almost surely strictly greater than the growth rate of each individual asset.

In the context of Theorem 2, the volatility of the price process appears to be the only cause for any completely mixed constant proportions strategy to grow at a rate strictly greater than $\rho$, the growth rate of each particular asset. This result contradicts conventional finance theory, where the volatility of asset prices is usually regarded as an impediment to financial growth. The result shows that in the present context volatility serves as an endogenous source of its acceleration.

\section{Proofs of the main results and their explanation}

We first observe that if a strategy $H$ is generated according to formula (2) by a sequence $\lambda_{1}, \lambda_{2}, \ldots$ of vectors of investment proportions, then

$$
\begin{gathered}
V_{t}=S_{t} h_{t}=\sum_{m=1}^{K} S_{t}^{m} h_{t-1}^{m}=\sum_{m=1}^{K} \frac{S_{t}^{m}}{S_{t-1}^{m}} S_{t-1}^{m} h_{t-1}^{m}= \\
\sum_{m=1}^{K} \frac{S_{t}^{m}}{S_{t-1}^{m}} \lambda_{t-1}^{m} S_{t-1} h_{t-1}=V_{t-1} \sum_{m=1}^{K} R_{t}^{m} \lambda_{t-1}^{m}=\left(R_{t} \lambda_{t-1}\right) V_{t-1}
\end{gathered}
$$

for each $t \geq 2$, and so

$$
V_{t}=\left(R_{t} \lambda_{t-1}\right)\left(R_{t-1} \lambda_{t-2}\right) \ldots\left(R_{2} \lambda_{1}\right) V_{1}, t \geq 2 .
$$

Proof of Theorem 1. By virtue of (9), we have

$$
V_{t}=\left[V_{1}\left(R_{1} \lambda\right)^{-1}\right]\left(R_{1} \lambda\right)\left(R_{2} \lambda\right) \ldots\left(R_{t} \lambda\right),
$$


and so

$$
\lim _{t \rightarrow \infty} \frac{1}{t} \ln V_{t}=\lim _{t \rightarrow \infty} \frac{1}{t} \sum_{j=1}^{t} \ln \left(R_{j} \lambda\right)=E \ln \left(R_{t} \lambda\right) \text { (a.s.) }
$$

by virtue of Birkhoff's ergodic theorem. It remains to show that if assumption $(\mathbf{V})$ holds, then $E \ln \left(R_{t} \lambda\right)>\sum_{k=1}^{K} \lambda^{k} \rho^{k}$. To this end observe that condition $(\mathbf{V})$ is equivalent to the following one:

(V1) For some $t \geq 1$ (and hence, by virtue of stationarity, for each $t \geq 1$ ), the probability

$$
P\left\{R_{t}^{k} \neq R_{t}^{m} \text { for some } 1 \leq k, m \leq K\right\}
$$

is strictly positive.

Indeed, we have $S_{t}^{k} / S_{t}^{m} \neq S_{t-1}^{k} / S_{t-1}^{m}$ if and only if $S_{t}^{k} / S_{t-1}^{k} \neq S_{t}^{m} / S_{t-1}^{m}$, which can be written as $R_{t}^{k} \neq R_{t}^{m}$. Denote by $\delta_{t}$ the random variable that is equal to 1 if the event $\left\{R_{t}^{k} \neq R_{t}^{m}\right.$ for some $\left.1 \leq k, m \leq K\right\}$ occurs and 0 otherwise. Condition (V) means that $P\left\{\max _{t \geq 1} \delta_{t}=1\right\}>0$, while (V1) states that, for some $t$ (and hence for each $t$ ), $P\left\{\delta_{t}=1\right\}>0$. The latter property is equivalent to the former because

$$
\left\{\max _{t \geq 1} \delta_{t}=1\right\}=\bigcup_{t=1}^{\infty}\left\{\delta_{t}=1\right\} .
$$

By using Jensen's inequality and (V1), we find that

$$
\ln \sum_{k=1}^{K} R_{t}^{k} \lambda^{k}>\sum_{k=1}^{K} \lambda^{k}\left(\ln R_{t}^{k}\right)
$$

with strictly positive probability, while the non-strict inequality holds always. Consequently,

$$
E \ln \left(R_{t} \lambda\right)>\sum_{k=1}^{K} \lambda^{k} E\left(\ln R_{t}^{k}\right)=\sum_{k=1}^{K} \lambda^{k} \rho^{k},
$$

which completes the proof.

The above considerations yield a rigorous proof of the fact of volatility induced growth. But what is the intuition, the underlying fundamental reason for it? We have only one explanation, which is nothing but a repetition in one phrase of the idea of the above proof. If $R_{t}^{1}, \ldots, R_{t}^{K}$ are random returns of assets $k=1,2, \ldots, K$, then the asymptotic growth rates of these 
assets are $E \ln R_{t}^{k}$, while the asymptotic growth rate of a constant proportions strategy is $E \ln \left(\sum \lambda^{k} R_{t}^{k}\right)$, which is strictly greater than $\sum \lambda^{k} E \ln \left(R_{t}^{k}\right)$ by Jensen's inequality - because the function $\ln x$ is strictly concave.

It would be nice, however, to give a general common-sense explanation of volatility-induced growth, without using such terms as a "strictly convex function," "Jensen's inequality," etc. One can, indeed, find in the literature explanations of examples of volatility pumping based on the following reasoning (see, e.g., Fernholz and Shay [11], Luenberger [21]). The reason for growth lies allegedly in the fact that constant proportions always force one to "buy low and sell high" - the common sense dictum of all trading. Those assets whose prices have risen from the last rebalance date will be overweighted in the portfolio, and their holdings must be reduced to meet the required proportions and to be replaced in part by assets whose prices have fallen and whose holdings must therefore be increased. Obviously, for this mechanism to work the prices must change in time; if they are constant, one cannot get any profit from trading.

We have, alas, repeated this reasoning ourselves (e.g. in [10] and in an earlier version of the present paper), but somewhat deeper reflection on this issue inevitably leads to the conclusion that the above argument does not explain everything and raises more questions than gives answers. For example, what is the meaning of "high" and "low"? If the price follows a geometric random walk, the set of its values is generally unbounded, and for every value there is a larger value. One can say that "high" and "low" should be understood in relative terms, based on the comparison of the prices today and yesterday. Fine, but what if the prices of all the assets increase or decrease simultaneously? Thus, the above argument, to be made valid, should be at least relativized, both with respect to time and the assets.

However, a more substantial lacuna in such reasoning is that it does not reflect the assumption of constancy of investment proportions. This leads to the question: what will happen if the "common sense dictum of all trading" is pushed to the extreme and the portfolio is rebalanced so as to sell all those assets that gain value and buy only those ones which lose it? Assume, for example, that there are two assets, the price $S_{t}^{1}$ of the first (riskless) is always 1 , and the price $S_{t}^{2}$ of the second (risky) follows a geometric random walk, so that the gross return on it can be either 2 or $1 / 2$ with equal probabilities. Suppose the investor sells the second asset and invests all wealth in the first if the price $S_{t}^{2}$ goes up and performs the converse operation, betting all wealth on the risky asset, if $S_{t}^{2}$ goes down. Then the sequence $\lambda_{t}=\left(\lambda_{t}^{1}, \lambda_{t}^{2}\right)$ of the vectors of investment proportions will be i.i.d. with values $(0,1)$ and $(1,0)$ taken on with equal probabilities. Furthermore, $\lambda_{t-1}$ will be independent of 
$R_{t}$. By virtue of (9), the growth rate of the portfolio value for this strategy is equal to $E \ln \left(R_{t} \lambda_{t-1}\right)=\left[\ln (0 \cdot 1+1 \cdot 2)+\ln \left(0 \cdot 1+1 \cdot \frac{1}{2}\right)+\ln (1 \cdot 1+0 \cdot 2)+\right.$ $\left.\ln \left(1 \cdot 1+0 \cdot \frac{1}{2}\right)\right] / 4=0$, which is the same as the growth rate of each of the two assets $k=1,2$ and is strictly less than the growth rate of any completely mixed constant proportions strategy.

\section{Stationary markets: puzzles and misconceptions}

Consider a market where the price process $S_{t}$ (and not only the process of asset returns $R_{t}$ ) is ergodic and stationary and where $E\left|\ln S_{t}^{k}\right|<\infty$. This situation is a special case of stationary returns, because if the vector process $S_{t}$ is stationary, then the process $R_{t}$ is stationary as well. In this case the growth rate of each asset is zero,

$$
E \ln R_{t}^{k}=E \ln S_{t}^{k}-E \ln S_{t-1}^{k}=0,
$$

while, as we have seen, any completely mixed constant proportions strategy grows at a strictly positive exponential rate. The assumption of stationarity of asset prices, perhaps after some detrending, seems plausible when modeling currency markets $[7,16]$. Then the "prices" are determined by exchange rates of all the currencies with respect to some selected reference currency.

We performed a casual experiment, asking numerous colleagues (in private, at seminars and at conferences) to promptly guess the correct answer to the following question.

Question 1. Suppose vectors of asset prices $S_{t}=\left(S_{t}^{1}, \ldots, S_{t}^{K}\right)$ fluctuate randomly, forming a stationary stochastic process (assume even that $S_{t}$ are i.i.d.). Consider a fixed-mix self-financing investment strategy prescribing rebalancing one's portfolio at each of the dates $t=1,2, \ldots$ so as to keep equal investment proportions of wealth in all the assets. What will happen with the portfolio value in the long run, as $t \rightarrow \infty$ ? What will be its tendency: (a) to decrease; (b) to increase; or (c) to fluctuate randomly, converging in one sense or another to a stationary process.

The audience of our respondents was quite broad and professional, but practically nobody succeeded in guessing the correct answer, (b). Among those who expressed a clear guess, nearly all selected (c). There were also a couple of respondents who decided to bet on (a).

Common intuition suggests that if the market is stationary, then the portfolio value $V_{t}$ for a constant proportions strategy must converge in one sense or another to a stationary process. The usual intuitive argument in support of this conjecture appeals to the self-financing property (3). The 
self-financing constraint seems to exclude possibilities of unbounded growth. This argument is also substantiated by the fact that in the deterministic case both the prices and the portfolio value are constant. This way of reasoning makes the answer (c) to the above question more plausible a priori than the others.

It might seem surprising that the wrong guess (c) has been put forward even by those who have known about examples of volatility pumping for a long time. The reason for this might lie in the non-traditional character of the setting where not only the asset returns but the prices themselves are stationary. Moreover, the phenomenon of volatility-induced growth is more paradoxical in the case of stationary prices, where growth emerges "from nothing." In the conventional setting of stationary returns, volatility serves as the cause of an acceleration of growth, rather than its emergence from prices with zero growth rates.

A typical way of understanding the correct answer to Question 1 is to reduce it to something well-known that is apparently relevant. A good candidate for this is the concept of arbitrage. Getting something from nothing as a result of an arbitrage opportunity seems to be similar to the emergence of growth in a stationary setting where there are no obvious sources for growth.

As long as we deal with an infinite time horizon, we would have to consider some kind of asymptotic arbitrage (e.g. Ross [27], Huberman [15], Kabanov and Kramkov [17], Klein and Schachermayer [19]). However, all known concepts of this kind are much weaker than what we would need in the present context. According to our results, growth is exponentially fast, unbounded wealth is achieved with probability one, and the effect of growth is demonstrated for specific (constant proportions) strategies. None of these properties can be directly deduced from asymptotic arbitrage.

Thus there are no convincing arguments showing that volatility-induced growth in stationary markets can be derived from, or explained by, asymptotic arbitrage over an infinite time horizon. But what can be said about relations between stationarity and arbitrage over finite time intervals? As is known, there are no arbitrage opportunities (over a finite time horizon) if and only if there exists an equivalent martingale measure. A stationary process can be viewed as an "antipodal concept" to the notion of a martingale. This might lead to the conjecture that in a stationary market arbitrage is a typical situation. Is this true or not? Formally, the question can be stated as follows.

Question 2. Suppose the process $S_{t}=\left(S_{t}^{1}, \ldots, S_{t}^{K}\right)$ of the vectors of 
asset prices is stationary, and moreover, assume that the vectors $S_{t}$ are i.i.d. Furthermore, suppose the first asset $k=1$ is riskless and its price $S_{t}^{1}$ is equal to one. Does this market have arbitrage opportunities over a finite time horizon?

When asking this question, we assume that the market is frictionless and that there are no portfolio constraints. In particular, all short sales are allowed. An arbitrage opportunity over a time horizon $t=0, \ldots, T$ is understood in the conventional sense. It means the existence of a selffinancing trading strategy $\left(h_{0}, \ldots, h_{T}\right)$ such that $S_{0} h_{0}=0, S_{T} h_{T} \geq 0$ a.s. and $P\left\{S_{T} h_{T}>0\right\}>0$.

The answer to this question, as well as to the previous one, is practically never guessed immediately. Surprisingly, the answer depends, roughly speaking, on whether the distribution of the random vector $\tilde{S}_{t}:=\left(S_{t}^{2}, \ldots, S_{t}^{K}\right)$ of prices of the risky assets is continuous or discrete. For example, if $\tilde{S}_{t}$ takes on a finite number of values, then an arbitrage opportunity exists. If the distribution of $\tilde{S}_{t}$ is continuous, there are no arbitrage opportunities. More precisely, the result is as follows. Let $G$ be the support of the distribution of the random vector $\tilde{S}_{t}$ (which is assumed to be non-degenerate) and let $F:=c l$ co $G$ be the closure of the convex hull of $G$. Denote by $\partial_{r} F$ the relative boundary of $F$, i.e. the boundary of the convex set $F$ in the smallest linear manifold containing $F$.

Theorem 3. If $P\left\{\tilde{S}_{t} \in \partial_{r} F\right\}=0$, then for any $T$ there are no arbitrage opportunities over the time horizon of length $T$. If $P\left\{\tilde{S}_{t} \in \partial_{r} F\right\}>0$, then for each $T$ there is an arbitrage opportunity over the time horizon of length $T$.

For a proof of this result see [9].

\section{Growth acceleration, volatility reduction and noise-induced stability}

The questions we analyze in this section stem from an example of volatility pumping considered originally by Fernholz and Shay [11] and later others (e.g. Luenberger [21]). The framework for this example is the well-known continuous-time model developed by Merton and others, in which the price processes $S_{t}^{k}(t \geq 0)$ of two assets $k=1,2$ are supposed to be solutions to the stochastic differential equations $d S_{t}^{k} / S_{t}^{k}=\mu_{k} d t+\sigma_{k} d W_{t}^{k}$, where the $W_{t}^{k}$ are independent (standard) Wiener processes and $S_{0}^{k}=1$. As is well-known, these equations admit explicit solutions $S_{t}^{k}=\exp \left[\mu_{k} t-\left(\sigma_{k}^{2} / 2\right) t+\sigma_{k} W_{t}^{k}\right]$. 
Given some $\theta \in(0,1)$, the value $V_{t}$ of the constant proportions portfolio prescribing investing the proportions $\theta$ and $1-\theta$ of wealth into assets $k=1,2$ is the solution to the equation

$$
d V_{t} / V_{t}=\left[\theta \mu_{1}+(1-\theta) \mu_{2}\right] d t+\theta \sigma_{1} d W_{t}^{1}+(1-\theta) \sigma_{2} d W_{t}^{2} .
$$

Equivalently, $V_{t}$ can be represented as the solution to the equation $d V_{t} / V_{t}=$ $\bar{\mu} d t+\bar{\sigma} d W_{t}$, where $\bar{\mu}:=\theta \mu_{1}+(1-\theta) \mu_{2}, \bar{\sigma}^{2}:=\left(\theta \sigma_{1}\right)^{2}+\left[(1-\theta) \sigma_{2}\right]^{2}$ and $W_{t}$ is a standard Wiener process. Thus, $V_{t}=\exp \left[\bar{\mu} t-\left(\bar{\sigma}^{2} / 2\right) t+\bar{\sigma} W_{t}\right]$, and so the growth rate and the volatility of the portfolio value process $V_{t}$ are given by $\bar{\mu}-\left(\bar{\sigma}^{2} / 2\right)$ and $\bar{\sigma}$. In particular, if $\mu_{1}=\mu_{2}=\mu$ and $\sigma_{1}=\sigma_{2}=\sigma$, then the growth rate and the volatility of $V_{t}$ are equal to

$$
\mu-\left(\bar{\sigma}^{2} / 2\right) \text { and } \bar{\sigma}=\sigma \sqrt{\theta^{2}+(1-\theta)^{2}}<\sigma,
$$

while for each individual asset the growth rate and the volatility are $\mu-$ $\left(\sigma^{2} / 2\right)$ and $\sigma$, respectively.

Thus, in this example, the use of a constant proportions strategy prescribing investing in a mixture of two assets leads (due to diversification) to an increase of the growth rate and to a simultaneous decrease of the volatility. When looking at the expressions in (13), the temptation arises even to say that the volatility reduction is the cause of volatility-induced growth. Indeed, the growth rate $\mu-\left(\bar{\sigma}^{2} / 2\right)$ is greater than the growth rate $\mu-\left(\sigma^{2} / 2\right)$ because $\bar{\sigma}<\sigma$. This suggests speculation along the following lines. Volatility is something like energy. When constructing a mixed portfolio, it converts into growth and therefore decreases. The greater the volatility reduction, the higher the growth acceleration.

Do such speculations have any grounds in the general case, or do they have a justification only in the above example? To formalize this question and answer it, let us return to the discrete time-framework we deal with in this paper. Suppose there are two assets with i.i.d. vectors of returns $R_{t}=\left(R_{t}^{1}, R_{t}^{2}\right)$. Let $(\xi, \eta):=\left(R_{1}^{1}, R_{1}^{2}\right)$ and assume, to avoid technicalities, that the random vector $(\xi, \eta)$ takes on a finite number of values and is strictly positive. The value $V_{t}$ of the portfolio generated by a fixed-mix strategy with proportions $x$ and $1-x(0<x<1)$ is computed according to the formula

$$
V_{t}=V_{1} \prod_{j=2}^{t}\left[x R_{j}^{1}+(1-x) R_{j}^{2}\right], t \geq 2
$$

(see (9)). The growth rate of this process and its volatility are given, respectively, by the expectation $E \ln \zeta_{x}$ and the standard deviation $\sqrt{\operatorname{Var} \ln \zeta_{x}}$ 
of the random variable $\ln \zeta_{x}$, where $\zeta_{x}:=\ln [x \xi+(1-x) \eta]$. We know from the above analysis that the growth rate increases when mixing assets with the same growth rate. What can be said about volatility? Specifically, let us consider the following question.

Question 3. (a) Suppose Var $\ln \xi=$ Var $\ln \eta$. Is it true that $\operatorname{Var} \ln [x \xi+$ $(1-x) \eta] \leq V$ ar $\ln \xi$ when $x \in(0,1)$ ? (b) More generally, is it true that Var $\ln [x \xi+(1-x) \eta] \leq \max (\operatorname{Var} \ln \xi, \operatorname{Var} \ln \eta)$ for $x \in(0,1)$ ?

Query (b) asks whether the logarithmic variance is a quasi-convex functional. Questions (a) and (b) can also be stated for volatility defined as the square root of logarithmic variance. They will have the same answers because the square root is a strictly monotone function. Positive answers to these questions would substantiate the above conjecture of volatility reduction-negative, refute it.

It turns out that in general (without additional assumptions on $\xi$ and $\eta$ ) the above questions $3(\mathrm{a})$ and $3(\mathrm{~b})$ have negative answers. To show this consider two i.i.d. random variables $U$ and $V$ with values 1 and $a>0$ realized with equal probabilities. Consider the function

$$
f(y):=\operatorname{Var} \ln [y U+(1-y) V], y \in[0,1] .
$$

By evaluating the first and the second derivatives of this function at $y=1 / 2$, one can show the following. There exist some numbers $0<a_{-}<1$ and $a_{+}>1$ such that the function $f(y)$ attains its minimum at the point $y=1 / 2$ when $a$ belongs to the closed interval $\left[a_{-}, a_{+}\right]$and it has a local maximum (!) at $y=1 / 2$ when $a$ does not belong to this interval. The numbers $a_{-}$ and $a_{+}$are given by

$$
a_{ \pm}=2 e^{4}-1 \pm \sqrt{\left(2 e^{4}-1\right)^{2}-1}
$$

where $a_{-} \approx 0.0046$ and $a_{+} \approx 216.388$. If $a \in\left[a_{-}, a_{+}\right]$, the function $f(y)$ is convex, but if $a \notin\left[a_{-}, a_{+}\right]$, its graph looks like then one depicted in Figure 1.

Fix any $a$ for which the graph of $f(y)$ is as in Figure 1. Consider any number $y_{0}<1 / 2$ which is greater than the smallest local minimum of $f(y)$ and define $\xi:=y_{0} U+\left(1-y_{0}\right) V$ and $\eta:=y_{0} V+\left(1-y_{0}\right) U$. ( $U$ and $V$ may be interpreted as "factors" on which the returns $\xi$ and $\eta$ on the two assets depend.) Then $\operatorname{Var} \ln [(\xi+\eta) / 2]>\operatorname{Var} \ln \xi=\operatorname{Var} \ln \eta$, which yields a negative answer both to (a) and (b). In this example $\xi$ and $\eta$ are dependent. It would be of interest to investigate questions (a) and (b) for general independent random variables $\xi$ and $\eta$. It can be shown that the answer to (b) is positive if one of the variables $\xi$ and $\eta$ is constant. But even in this case 


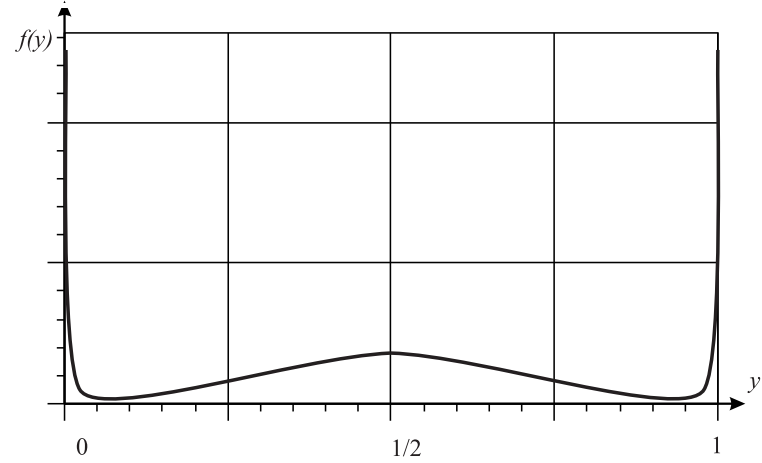

Figure 1: Graph of the function $f(y)$ in eq. (14) for $a=10^{4}$.

the function $\operatorname{Var} \ln [x \xi+(1-x) \eta]$ is not necessarily convex: it may have an inflection point in $(0,1)$, which can be easily shown by examples involving two-valued random variables.

Thus it can happen that a mixed portfolio may have a greater volatility than each of the assets from which it has been constructed. Consequently, the above conjecture and the "energy interpretation" of volatility are generally not valid. It is interesting, however, to find additional conditions under which assertions regarding volatility reduction hold true. In this connection, we can assert the following fact.

Theorem 4. Let $U$ and $V$ be independent random variables bounded above and below by strictly positive constants. If $U$ is not constant, then Var $\ln [y U+(1-y) V]<$ Var $\ln U$ for all $y \in(0,1)$ sufficiently close to 1.

Volatility can be regarded as a quantitative measure of instability of the portfolio value. The above result shows that small independent noise can reduce volatility. This result is akin to a number of known facts about noise-induced stability, e.g., Abbott [1] and Mielke [23]. An analysis of links between the topic of the present work and results about stability under random noise might constitute an interesting theme for further research.

Proof of Theorem 4 can be obtained by evaluating the derivative of the function $f(y)$ defined by (14) at $y=1$. Basic computations show that

$$
f^{\prime}(1)=2(E V)\left(-E e^{-Z} Z+E e^{-Z} \cdot E Z\right)
$$

where $Z=\ln U$. The assertion of the theorem is valid because $f^{\prime}(1)>0$. The verification of this inequality is based on the following fact. If $\phi(z)$ is a function on $(-\infty,+\infty)$ with $\phi^{\prime}(z)>0$, then

$$
E[Z \phi(Z)]>(E Z) E \phi(Z)
$$


for any non-constant bounded random variable $Z$. This fact follows from Jensen's inequality applied to the strictly convex function $\psi(x):=\int_{0}^{x} \phi(z) d z$ and from the relation $\psi(y)-\psi(x) \geq \phi(x)(y-x)$ (to obtain (16) put $x:=Z$, $y:=E Z$ and take the expectation). By applying (16) to $\phi(z):=-e^{-z}$, we find that the expression in (15) is positive.

\section{Volatility-induced growth under small transac- tion costs}

We now assume that, in the market under consideration (see Section 2), there are transaction costs for buying and selling assets. When selling $x$ units of asset $k$ at time $t$, one receives the amount $\left(1-\varepsilon_{-}^{k}\right) S_{t}^{k} x$ (rather than $S_{t}^{k} x$ as in the frictionless case). To buy $x$ units of asset $k$, one has to pay $\left(1+\varepsilon_{+}^{k}\right) S_{t}^{k} x$. The numbers $\varepsilon_{-}^{k}, \varepsilon_{+}^{k} \geq 0, k=1,2, \ldots, K$ (the transaction cost rates) are assumed given. In this market, a trading strategy $H=\left(h_{0}, h_{1}, \ldots\right)$ is self-financing if

$$
\sum_{k=1}^{K}\left(1+\varepsilon_{+}^{k}\right) S_{t}^{k}\left(h_{t}^{k}-h_{t-1}^{k}\right)_{+} \leq \sum_{k=1}^{K}\left(1-\varepsilon_{-}^{k}\right) S_{t}^{k}\left(h_{t-1}^{k}-h_{t}^{k}\right)_{+}, t \geq 1,
$$

where $x_{+}=\max \{x, 0\}$. Inequality (17) means that asset purchases can be made only at the expense of asset sales.

Relation (17) is equivalent to

$$
\sum_{k=1}^{K} S_{t}^{k}\left(h_{t}^{k}-h_{t-1}^{k}\right) \leq-\sum_{k=1}^{K} \varepsilon_{+}^{k} S_{t}^{k}\left(h_{t}^{k}-h_{t-1}^{k}\right)_{+}-\sum_{k=1}^{K} \varepsilon_{-}^{k} S_{t}^{k}\left(h_{t-1}^{k}-h_{t}^{k}\right)_{+}
$$

(which follows from the identity $x_{+}-(-x)_{+}=x$ ). Therefore, if the portfolio $h_{t}$ differs from the portfolio $h_{t-1}$ in at least one position $k$ for which $\varepsilon_{+}^{k} \neq 0$ and $\varepsilon_{-}^{k} \neq 0$, then $S_{t} h_{t}<S_{t} h_{t-1}$. Thus, under transaction costs, portfolio rebalancing typically leads to a loss of wealth. The number $S_{t} h_{t} / S_{t} h_{t-1} \leq 1$ is called the loss coefficient (of the strategy $H$ at time $t$ ).

We say that $H=\left(h_{0}, h_{1}, \ldots\right)$ is a constant proportions strategy with vector of proportions $\lambda=\left(\lambda^{1}, \ldots, \lambda^{K}\right) \in \Delta$ if $S_{t}^{k} h_{t}^{k}=\lambda^{k} S_{t} h_{t}$ for all $k=$ $1,2, \ldots, K$ and $t=1,2, \ldots$ (cf. (4)). Let $\delta \in(0,1)$ be a constant. Given a vector of proportions $\lambda=\left(\lambda^{1}, \ldots, \lambda^{K}\right) \in \Delta$ and a non-zero initial portfolio $h_{0} \geq 0$, define recursively

$$
h_{t}^{k}=(1-\delta) \lambda^{k} S_{t} h_{t-1} / S_{t}^{k}(k=1,2, \ldots, K, t=1,2, \ldots) .
$$


This rule defines a trading strategy with constant investment proportions $\lambda^{1}, \ldots, \lambda^{K}$ and a constant loss coefficient $1-\delta$. We will call it the $(\delta, \lambda)$ strategy.

Theorem 5 below extends the results of Theorems 1 and 2 to the model with transaction costs. As before, we assume that hypotheses $(\mathbf{R})$ and $(\mathbf{V})$ hold.

Theorem 5. Let $\lambda=\left(\lambda^{1}, \ldots, \lambda^{K}\right) \in \Delta$ be a strictly positive vector. If $\delta \in(0,1)$ is small enough, then the $(\delta, \lambda)$-strategy $H$ defined by (18) has a growth rate strictly greater than $\sum_{k=1}^{K} \lambda^{k} \rho^{k}$ (a.s.), and so if $\rho^{1}=\ldots=$ $\rho^{K}=\rho$, then the growth rate of $H$ is strictly greater than $\rho$ (a.s.). Further, if the transaction cost rates $\varepsilon_{-}^{k}, \varepsilon_{+}^{k} \geq 0, k=1,2, \ldots, K$, are small enough (in particular, if they do not exceed $\delta / 2$ ), then the strategy $H$ is self-financing.

The purpose of this theorem is to demonstrate that the results on volatilityinduced growth remain valid under small transaction costs. In contrast with a number of the questions we considered previously, the answer to the question we pose here is quite predictable and does not contradict intuition. We deal in Theorem 5 with constant proportions strategies of a special formthose for which the loss rate is constant (and small enough). We are again not concerned with the question of optimality of such strategies for one criterion or another.

Proof of Theorem 5. We first observe that the growth rate of the strategy $H$ is equal to $E \ln \left[(1-\delta) R_{t} \lambda\right]$. This fact is proved exactly like (11) (multiply the vectors of proportions in (8), (9), (10) and (11) by $(1-\delta))$. According to (12), we have $E \ln \left(R_{t} \lambda\right)>\sum_{k=1}^{K} \lambda^{k} \rho^{k}$. This inequality will remain valid if $\lambda$ is replaced by $(1-\delta) \lambda$, provided $\delta \in(0,1)$ is small enough. Fix any such $\delta \in(0,1)$. Denote by $\varepsilon$ the greatest of the numbers $\varepsilon_{-}^{k}, \varepsilon_{+}^{k}$. It remains to show that $H$ is self-financing when $\varepsilon \leq \delta / 2$. To this end we note that inequality (17) is implied by

$$
\sum_{k=1}^{K}(1+\varepsilon) S_{t}^{k}\left(h_{t}^{k}-h_{t-1}^{k}\right)_{+} \leq \sum_{k=1}^{K}(1-\varepsilon) S_{t}^{k}\left(h_{t-1}^{k}-h_{t}^{k}\right)_{+},
$$

which is equivalent to

$$
\varepsilon \sum_{k=1}^{K}\left|S_{t}^{k} h_{t}^{k}-S_{t}^{k} h_{t-1}^{k}\right| \leq S_{t}\left(h_{t-1}-h_{t}\right) .
$$

In view of (18), the right-hand side of the last inequality is equal to $\delta S_{t} h_{t-1}$, 
and the left-hand side can be estimated as follows:

$$
\begin{gathered}
\varepsilon \sum_{k=1}^{K}\left|(1-\delta) \lambda^{k} S_{t} h_{t-1}-S_{t}^{k} h_{t-1}^{k}\right| \leq \varepsilon \sum_{k=1}^{K}(1-\delta) \lambda^{k} S_{t} h_{t-1}+\varepsilon \sum_{k=1}^{K} S_{t}^{k} h_{t-1}^{k} \\
=\varepsilon(1-\delta) S_{t} h_{t-1}+\varepsilon S_{t} h_{t-1} \leq 2 \varepsilon S_{t} h_{t-1} .
\end{gathered}
$$

Consequently, if $0 \leq \varepsilon \leq \delta / 2$, then the strategy $H$ is self-financing.

\section{Growth under transaction costs: an example}

In this section we consider an example (a binomial model) in which quantitative estimates for the size of the transaction costs needed for the validity of the result on volatility-induced growth can be provided. Suppose that there are two assets $k=1,2$ : one riskless and one risky. The price of the former is constant and equal to 1 . The price of the latter follows a geometric random walk. It can either jump up by $u>1$ or down by $u^{-1}$ with equal probabilities. Thus both securities have zero growth rates.

Suppose the investor pursues the constant proportions strategy prescribing to keep $50 \%$ of wealth in each of the securities. There are no transaction costs for buying and selling the riskless asset, and the transaction cost rate for buying and selling the risky asset is $\varepsilon \in[0,1)$. Assume the investor's portfolio at time $t-1$ contains $v$ units of cash; then the value of the risky position of the portfolio must be also equal to $v$. At time $t$, the riskless position of the portfolio will remain the same, and the value of the risky position will become either $u v$ or $u^{-1} v$ with equal probability. In the former case, the investor rebalances his/her portfolio by selling an amount of the risky asset worth $A$ so that

$$
v+(1-\varepsilon) A=v u-A .
$$

By selling an amount of the risky asset of value $A$ in the current prices, the investor receives $(1-\varepsilon) A$, and this sum of cash is added to the riskless position of the portfolio. After rebalancing, the values of both portfolio positions must be equal, which is expressed in (20). From (20) we obtain $A=v(u-1)(2-\varepsilon)^{-1}$. The positions of the new (rebalanced) portfolio, measured in terms of their current values, are equal to $v+(1-\varepsilon) A=v[1+$ $(1-\varepsilon)(2-\varepsilon)^{-1}(u-1)$ ]. In the latter case (when the value of the risky position becomes $u^{-1} v$ ), the investor buys some amount of the risky asset worth $B$, for which the amount of cash $(1+\varepsilon) B$ is needed, so that

$$
v-(1+\varepsilon) B=u^{-1} v+B .
$$


From this, we find $-B=v\left(u^{-1}-1\right)(2+\varepsilon)^{-1}$, and so $v-(1+\varepsilon) B=$ $v\left[1+(1+\varepsilon)(2+\varepsilon)^{-1}\left(u^{-1}-1\right)\right]$.

Thus, the portfolio value at each time $t$ is equal to its value at time $t-1$ multiplied by the random variable $\xi$ such that $P\left\{\xi=g^{\prime}\right\}=P\left\{\xi=g^{\prime \prime}\right\}=$ $1 / 2$, where $g^{\prime}:=1+(1+\varepsilon)(2+\varepsilon)^{-1}\left(u^{-1}-1\right)$ and $g^{\prime \prime}:=1+(1-\varepsilon)(2-$ $\varepsilon)^{-1}(u-1)$. Consequently, the asymptotic growth rate of the portfolio value, $E \ln \xi=(1 / 2)\left(\ln g^{\prime}+\ln g^{\prime \prime}\right)$, is equal to $(1 / 2) \ln \phi(\varepsilon, u)$, where

$$
\phi(\varepsilon, u):=\left[1+(1+\varepsilon) \frac{u^{-1}-1}{2+\varepsilon}\right]\left[1+(1-\varepsilon) \frac{u-1}{2-\varepsilon}\right] .
$$

We have $E \ln \xi>0$, i.e., the phenomenon of volatility induced growth takes place, if $\phi(\varepsilon, u)>1$. For $\varepsilon \in[0,1)$, this inequality turns out to be equivalent to the following very simple relation: $0 \leq \varepsilon<(u-1)(u+1)^{-1}$. Thus, given $u>1$, the asymptotic growth rate of the fixed-mix strategy under consideration is greater than zero if the transaction cost rate $\varepsilon$ is less than $\varepsilon^{*}(u):=(u-1)(u+1)^{-1}$. We call $\varepsilon^{*}(u)$ the threshold transaction cost rate. Volatility-induced growth takes place when $0 \leq \varepsilon<\varepsilon^{*}(u)$.

The volatility $\sigma$ of the risky asset under consideration (the standard deviation of its logarithmic return) is equal to $\ln u$. In the above considerations, we assumed that $\sigma$-or equivalently, $u$-is fixed, and we examined $\phi(\varepsilon, u)$ as a function of $\varepsilon$. Let us now examine $\phi(\varepsilon, u)$ as a function of $u$ when the transaction cost rate $\varepsilon$ is fixed and strictly positive. For the derivative of $\phi(\varepsilon, u)$ with respect to $u$, we have

$$
\phi_{u}^{\prime}(\varepsilon, u)=\frac{1+\varepsilon}{4-\varepsilon^{2}}\left[\frac{1-\varepsilon}{1+\varepsilon}-u^{-2}\right] .
$$

If $u=1$, then $\phi_{u}^{\prime}(\varepsilon, 1)<0$. Thus if the volatility of the risky asset is small, the performance of the constant proportions strategy at hand is worse than the performance of each individual asset. This fact refutes the possible conjecture that "the higher the volatility, the higher the induced growth rate." Further, the derivative $\phi_{u}^{\prime}(\varepsilon, u)$ is negative when $u \in\left[0, u_{*}(\varepsilon)\right)$, where $u_{*}(\varepsilon):=(1-\varepsilon)^{-1 / 2}(1+\varepsilon)^{1 / 2}$. For $u=u_{*}(\varepsilon)$ the asymptotic growth rate of the constant proportions strategy at hand attains its minimum value. For those values of $u$ which are greater than $u_{*}(\varepsilon)$, the growth rate increases, and it tends to infinity as $u \rightarrow \infty$. Thus, although the assertion "the greater the volatility, the greater the induced growth rate" is not valid always, it is valid (in the present example) under the additional assumption that the volatility is large enough. 


\section{Conclusion}

In this paper we have established the surprising result that when asset returns are stationary ergodic, their volatility, together with any fixed-mix trading strategy, generates a portfolio growth rate in excess of the individual asset growth rates. As a consequence, even if the growth rates of the individual securities all have mean zero, the value of a fixed-mix portfolio tends to infinity with probability one. By contrast with the twenty five years in which the effects of "volatility pumping" have been investigated in the literature by example, our results are quite general. They are obtained under assumptions which accommodate virtually all the empirical market return properties discussed in the literature. We have in this paper also dispelled the notion that the demonstrated acceleration of portfolio growth is simply a matter of "buying lower and selling higher." The example of Section 3 shows that our result depends critically on rebalancing to an arbitrary fixed mix of portfolio proportions. Any such mix defines the relative magnitudes of individual asset returns realized from volatility effects. This observation and our analysis of links between growth, arbitrage and noise-induced stability suggest that financial growth driven by volatility is a subtle and delicate phenomenon.

\section{References}

[1] Аввотт, D. (2001): "Overview: Unsolved problems of noise and fluctuations," Chaos, 11, 526-538.

[2] Algoet, P.H., And T.M. Cover (1988): "Asymptotic optimality and asymptotic equipartition properties of log-optimum investment," Annals of Probability, 16, 876-898.

[3] Aurell, E., R. Baviera, O. Hammarlid, M. Serva, and A. VulPiAni (2000): "A general methodology to price and hedge derivatives in incomplete markets," International Journal of Theoretical and Applied Finance, 3, 1-24.

[4] Billingsley, P. (1965): Ergodic Theory and Information. Wiley, New York.

[5] Breiman, L. (1961): "Optimal gambling systems for favorable games," In Fourth Berkeley Symposium on Mathematical Statistics and Probability, v. 1, pp. 65-78. 
[6] Cover, T.M. (1991) "Universal portfolios," Mathematical Finance, 1, $1-29$.

[7] Dempster, M.A.H., I.V. Evstigneev, and K.R. Schenk-Hoppé (2003): "Exponential growth of fixed-mix strategies in stationary asset markets," Finance and Stochastics, 7, 263-276.

[8] Dries, D., A. Ilhan, J. Mulvey, K.D. Simsek, and R. Sircar (2002): "Trend-following hedge funds and multi-period asset allocation," Quantitative Finance, 2, 354-361.

[9] Evstigneev, I.V., And D. Kapoor (2006): Arbitrage in stationary markets, Discussion Paper 0608, School of Economic Studies, University of Manchester.

[10] Evstigneev, I.V., And K.R. Schenk-Hoppé (2002): "From rags to riches: On constant proportions investment strategies," International Journal of Theoretical and Applied Finance, 5, 563-573.

[11] Fernholz, R., And B. Shay (1982): "Stochastic portfolio theory and stock market equilibrium," Journal of Finance, 37, 615-624.

[12] Föllmer, H., ANd A. Schied (2002): Stochastic Finance - An Introduction in Discrete Time. Walter de Gruyter, Berlin.

[13] Hakansson, N.H., And W.T. Ziemba (1995): "Capital growth theory." In Handbooks in Operations Research and Management Science, Volume 9, Finance, R.A. Jarrow, V. Maksimovic and W.T. Ziemba, eds., Elsevier, Amsterdam, pp. 65-86.

[14] Harmer, G.P., And D. Abbott, (1999): "Parrondo's paradox," Statistical Science, 14, 206-213.

[15] Huberman, G. (1982): "A simple approach to Arbitrage Pricing Theory," Journal of Economic Theory, 28, 183-191.

[16] Kabanov, Yu.M. (1999): "Hedging and liquidation under transaction costs in currency markets," Finance and Stochastics, 3, 237-248.

[17] Kabanov, Yu.M. and D.A. Kramkov (1994): "Large financial markets: Asymptotic arbitrage and contiguity," Theory of Probability and its Applications, 39, 222-228.

[18] KelLy, J.L. (1956): A new interpretation of information rate, Bell System Technical Journal, 35, 917-926. 
[19] Klein, I., And W. Schachermayer (1996): "Asymptotic arbitrage in non-complete large financial markets," Theory of Probability and its Applications, 41, 927-334.

[20] LI, Y. (1998): "Growth-security investment strategy for long and short runs," Management Science, 39, 915-924.

[21] Luenberger, D. (1998): Investment Science. Oxford University Press, New York.

[22] Maclean, L.C., W.T. Ziemba, and G. Blazenko (1992): "Growth versus security in dynamic investment analysis," Management Science, $38,1562-1585$.

[23] Mielke, A. (2000): "Noise induced stability in fluctuating bistable potentials," Physical Review Letters, 84, 18-821.

[24] Mulvey, J.M. (2001): "Multi-period stochastic optimization models for long-term investors." In Quantitative Analysis in Financial Markets, Vol. 3., M. Avellaneda, ed., World Scientific, Singapore, pp. 66-85.

[25] Mulvey, J.M., And W.T. Ziemba (eds.) 1998: Worldwide Asset and Liability Modeling, Cambridge, UK, Cambridge University Press.

[26] Perold, A.F., And W.F. Sharpe (1995): "Dynamic strategies for asset allocation," Financial Analysts Journal, 51, 149-160.

[27] Ross, S.A. (1976): "The arbitrage theory of asset pricing," Journal of Economic Theory, 13, 341-360. 